\title{
A New Phage Cocktail Against Multidrug, ESBL-Producer Isolates of Shigella sonnei and Shigella flexneri with Highly Efficient Bacteriolytic Activity
}

\author{
Khashayar Shahin, ${ }^{1,2}$ Majid Bouzari, ${ }^{1}$ Majid Komijani, ${ }^{3}$ and Ran Wang ${ }^{2,4}$
}

\begin{abstract}
The globally increasing incidence of antibiotic resistance in pathogenic microorganisms such as Shigella, a cause of human acute gastrointestinal infections, calls for developing effective alternatives. In this study, the antibiotic resistance pattern, extended-spectrum $\beta$-lactamase (ESBL)-production, and molecular characteristics of 70 multidrug-resistant isolates belong to the two most frequent species of Shigella genus, that is, Shigella sonnei (44 isolates) and Shigella flexneri (26 isolates) were investigated. These isolates were used to evaluate both specificity and activity of Shigella-specific bacteriophages, vB_SflS-ISF001, vB_SsoS-ISF002, and a cocktail of both. Twelve out of the 21 tested resistance genes were detected in the isolates. About 59\% of $S$. sonnei and $46 \%$ of $S$. flexneri isolates were identified as ESBL producers. The bacteriophages showed a high efficiency of plating (EOP $\geq 0.5$ ) in about $75 \%$ of the isolates. Moreover, the growth of $>85 \%$ of the isolates was inhibited by the phage cocktail of vB_SflS-ISF001 and vB_SsoS-ISF002. The phage cocktail was effective against a wide range of ESBL-positive and -negative isolates of $S$. sonnei and $S$. flexneri. Therefore, this phage cocktail has the potential to inhibit or significantly decrease the spread of drug-resistant Shigella in humans, food chains, and water/wastewater sanitation systems.
\end{abstract}

Keywords: shigellosis, Shigella spp., multidrug resistance, bacteriophage, phage therapy

\section{Introduction}

$S$ HIGELLA IS A GENUS of Gram-negative bacteria belonging to the Enterobacteriaceae family and includes four species, which can cause an acute form of gastrointestinal infection named bloody diarrhea or shigellosis. ${ }^{1}$ The global estimation is that at least 80 million bloody diarrhea cases occur annually with about 700,000 mortalities. ${ }^{2}$ Investigations show that about $70 \%$ of all the cases and $60 \%$ of the mortalities happen in children under the age of 5 years. $^{2}$ Due to two serious risk factors, the easy fecal/oral transmission of Shigella (direct contact with the infected individuals or consuming contaminated foods and water) and the low infectious dose $(<200$ bacteria), several outbreaks have been reported all over the world. ${ }^{2,3}$

The rapid spreading of antibiotic-resistant phenotypes among microorganisms is a global critical health issue as a consequence of abuse, inappropriate, and extensive use of antimicrobial agents against bacteria. ${ }^{4}$ In 2013, the Centers for Disease Control and Prevention announced that human being stands in the postantibiotic era, ${ }^{5}$ however, just a few years later (2017), World Health Organization (WHO) released a list of pathogens with an urgent need for new antibiotics or alternative control/treatment approaches. This list categorized the pathogens into three main groups. The extended-spectrum $\beta$-lactamase-producing (ESBL-producer) Enterobacteriaceae, responsible for at least 26,000 health care-associated infections and 1,700 death each year, were placed in the critical conditions group. ${ }^{4,6}$ In the case of Shigella species, their resistance rate to the available antimicrobial agents dramatically increased over the past 20 years (1999-2019). The National Antimicrobial Resistance Monitoring System (NARMS) of CDC (November 25, 2019) reported that in the United States, the year 1999, only $1 \%$, $0.3 \%, 1.6 \%, 56.4 \%$, and $57 \%$ of Shigella isolates were the resistance phenotypes to ceftriaxone, ciprofloxacin, nalidixic acid, streptomycin, and tetracycline, respectively, while in recent years these rates increased to $10.8 \%$ (ceftriaxone), $23.1 \%$ (ciprofloxacin), 98.3\% (nalidixic acid), 57\% (streptomycin), and $87 \%$ (tetracycline). ${ }^{5}$

Moreover, antibiotic-resistant infections are economic stress and a critical health issue that increase hospitalization

\footnotetext{
${ }^{1}$ Department of Biology, Faculty of Sciences, University of Isfahan, Isfahan, Iran.

${ }^{2}$ State Key Laboratory Cultivation Base of MOST, Institute of Food Safety and Nutrition, Jiangsu Academy of Agricultural Sciences, Nanjing, P.R. China.

${ }^{3}$ Department of Biology, Faculty of Science, Arak University, Arak, Iran.

${ }^{4}$ Jiangsu University, Zhenjiang, P.R. China.
} 
periods and decrease the chance of successful treatment. Thus, the discovery and development of alternative treatment approaches to reduce antibiotics consumption, and the emergence rate of resistant isolates is imperative to shorten the treatment duration and to enhance the success rate of treatment. A promising alternative for such a vital task is lytic bacteriophages (phage therapy). ${ }^{7,8}$ Bacteriophages are bacterial viruses with a natural tendency toward specific bacteria despite their resistance or susceptibility toward antibiotics. The specific nature of phage/bacteria interaction makes bacteriophages safe and suitable for use without any concerning about the side effects on other bacteria, in particular, the normal microbiota of a host organism. ${ }^{7-9}$

The current study aimed to disclose the antibiotic resistance pattern, resistance genes profile, and the integron genes among multidrug resistance (MDR) isolates of Shigella sonnei and Shigella flexneri. The second objective was to evaluate the lytic activity of vB_SsoS-ISF002, vB_SflSISF001, and a cocktail of both phages against ESBLproducing isolates of Shigella.

\section{Materials and Methods}

\section{Samples collection}

From January 2015 to September 2016, a number of MDR isolates of $S$. sonnei and $S$. flexneri were obtained from several medical centers and diagnostic laboratories in different provinces of Iran, including Isfahan, Fars, Hormozgan, and Kohkiloyeh va BoyerAhmad. To confirm the identity of the isolates, standard biochemical tests, including triple sugar

Table 1. Antibiotic Resistance Genes, Integrons, wbG $Z$, and RFC Primers

\begin{tabular}{|c|c|c|c|c|}
\hline Primer & Sequence $\left(5^{\prime}\right.$ to $\left.3^{\prime}\right)$ & Amplicon size $(b p)$ & Annealing $\left({ }^{\circ} \mathrm{C}\right)$ & Reference \\
\hline \multirow[t]{2}{*}{ wbgZ } & F:TCTGAATATGCCCTCTACGCT & 430 & 60 & 15 \\
\hline & R:GACAGAGCCCGAAGAACCG & & & \\
\hline \multirow[t]{2}{*}{$r f c$} & F:TTTATGGCTTCTTTGTCGGC & 537 & 60 & 15 \\
\hline & R:CTGCGTGATCCGACCATG & & & \\
\hline \multirow[t]{2}{*}{$S H V$} & F:GATGAACGCTTTCCCATGATG & 214 & 61 & 17 \\
\hline & R:CGCTGTTATCGCTCATGGTAA & & & \\
\hline \multirow[t]{2}{*}{$T E M$} & F:ATGAGTATTCAACATTTCCG & 867 & 58 & 17 \\
\hline & R:CTGACAGTTACCAATGCTTA & & & \\
\hline \multirow[t]{2}{*}{$C T X-M$} & F:TTTGCGATGTGCAGTACCAGTAA & 590 & 60 & 17 \\
\hline & R: CGATATCGTTGGTGGTGCCATA & & & \\
\hline \multirow[t]{2}{*}{$O X A$} & F:GCGTGGTTAAGGATGAACAC & 438 & 57 & 18 \\
\hline & R:CATCAAGTTCAACCCAACCG & & & \\
\hline \multirow[t]{2}{*}{ PER } & F:ATGAATGTCATTATAAAAGC & 925 & 59 & 20 \\
\hline & R:AATTTGGGCTTAGGGCAGAA & & & \\
\hline \multirow[t]{2}{*}{$V E B$} & F:CGACTTCCATTTCCCGATGC & 643 & 58 & 16 \\
\hline & R:GGACTCTGCAACAAATACGC & & & \\
\hline \multirow[t]{2}{*}{ GES } & F:ATGCGCTTCATTCACGCAC & 864 & 58 & 18 \\
\hline & R:CTATTTGTCCGTGCTCAGG & & & \\
\hline \multirow[t]{2}{*}{$C M Y$} & F:GACAGCCTCTTTCTCCACA & 1,010 & 61 & 14 \\
\hline & R:TGGAACGAAGGCTACGTA & & & \\
\hline \multirow[t]{2}{*}{$K P C$} & F:GTCTAGTTCTGCTGTCTTG & 798 & 59 & 19 \\
\hline & R:CTTGTCATCCTTGTTAGGCG & & & \\
\hline \multirow[t]{2}{*}{$N D M$} & F:GGTTTGGCGATCTGGTTTTC & 621 & 59 & 19 \\
\hline & R:GGAATGGCTCATCACGATC & & & \\
\hline \multirow[t]{2}{*}{ IMP } & F:CATGGTTTGGTGGTTCTTGT & 448 & 60 & 19 \\
\hline & R:ATAATTTGGCGGACTTTGGC & & & \\
\hline \multirow[t]{2}{*}{$q n r A$} & F:ATTTCTCACGCCAGGATTTG & 519 & 60 & 14 \\
\hline & R: GATCGGCAAAGGTTAGGTCA & & & \\
\hline \multirow[t]{2}{*}{$q n r B$} & F:GATCGTGAAAGCCAGAAAGG & 469 & 60 & 14 \\
\hline & R:ACGATGCCTGGTAGTTGTCC & & & \\
\hline \multirow[t]{2}{*}{$q n r S$} & F:ACGACATTCGTCAACTGCAA & 417 & 60 & 14 \\
\hline & R:TAAATTGGCACCCTGTAGGC & & & \\
\hline \multirow[t]{2}{*}{ aac } & F:TTGCGATGCTCTATGAGTGGCTA & 482 & 60 & 14 \\
\hline & R:CTCGAATGCCTGGCGTGTTT & & & \\
\hline \multirow[t]{2}{*}{ tetA } & F: GTGAAACCCAACATACCCC & 888 & 58 & 12 \\
\hline & R: GAAGGCAAGCAGGATGTAG & & & \\
\hline \multirow[t]{2}{*}{ tet $B$} & F:CCTTATCATGCCAGTCTTGC & 659 & 60 & 12 \\
\hline & R:ACTGCCGTTTTTTCGCC & & & \\
\hline \multirow[t]{2}{*}{ tetC } & F:CTTGAGAGCCTTCAACCCAG & 418 & 58 & 12 \\
\hline & R:ATGGTCGTCATCTACCTGCC & & & \\
\hline \multirow[t]{2}{*}{$\operatorname{tet} D$} & F:TGGGCAGATGGTCAGATAAG & 787 & 59 & 12 \\
\hline & R:CAGCACACCCTGTAGTTTTC & & & \\
\hline \multirow[t]{2}{*}{ intl } & 5'-CS:GGCATCCAAGCAGCAAG & Variable & 57 & 14 \\
\hline & 3'-CS AAGCAGACTTGACCTGA & & & \\
\hline int 2 & $\begin{array}{l}\text { hep74:CGGGATCCCGGACGGCATGCACGATTTGTA } \\
\text { hep51:GATGCCATCGCAAGTACGAG }\end{array}$ & Variable & 57 & 14 \\
\hline
\end{tabular}


iron, urease, motility, citrate utilization, oxidase, indole production, methyl red, and Voges-Proskauer (Merck, Germany) were performed on the isolates. ${ }^{10,11}$ Finally, the identity of each isolate was validated using specific primers for the amplification of $w b g Z$ (S. sonnei) and $r f c$ (S. flexneri) genes (Table 1). The standard slide agglutination method was done for serogrouping (Baharafshan Institute of Research \& Development, Iran). ${ }^{12}$ Standard strains of $S$. sonnei (ATCC 9290), S. flexneri (ATCC 12022), S. dysenteriae (persian type culture collection [PTCC] 1188), S. boydii (PTCC 9207), and E. coli (ATCC 25922) were used as the controls.

\section{Antimicrobial Susceptibility and extended-spectrum $\beta$-lactamase producer identification}

The antibiotic resistance pattern of all isolates was tested against 10 antibiotics from 5 different antibiotic groups (Oxoid, UK), including ampicillin $(25 \mu \mathrm{g})$, cefuroxime $(30 \mu \mathrm{g})$, ceftazidime $(30 \mu \mathrm{g})$, cefotaxime $(30 \mu \mathrm{g})$, cefepime $(30 \mu \mathrm{g})$, nalidixic acid $(30 \mu \mathrm{g})$, ciprofloxacin $(5 \mu \mathrm{g})$, tetracycline $(30 \mu \mathrm{g})$, streptomycin $(30 \mu \mathrm{g})$, and chloramphenicol $(30 \mu \mathrm{g})$ by the Kirby/Bauer disk diffusion method following the Clinical and Laboratory Standards Institute (CLSI) guidelines. ${ }^{13}$ After overnight incubation at $37^{\circ} \mathrm{C}$, the diameter of the zone of inhibition was measured, and the isolates were classified into three groups of resistant (R), intermediate (I), and susceptible (S) according to CLSI guideline. The isolates that showed resistance phenotype against at least three antibiotic groups were identified as MDR. ${ }^{1}$

The minimum inhibitory concentration of imipenem (Liofilchem, Italy) was measured using the $E$-test method following CLSI guidelines. ${ }^{13}$ The ESBL producer isolates were identified with the combined antibiotic disks method using ceftazidime $(30 \mu \mathrm{g})$ and ceftazidime $(30 \mu \mathrm{g})+$ clavulanic acid $(10 \mu \mathrm{g})$ (Rosco, Denmark) according to the CLSI guidelines. ${ }^{13}$ Where compared with the result of the ceftazidime disc (clavulanic acid), a $\geq 5 \mathrm{~mm}$ increase in the inhibition zone of the combination disc (with clavulanic acid) was considered as ESBL producer isolate. E.coli (ATCC
25922), Staphylococcus aureus (ATCC 25923), and Klebsiella pneumonia (ATCC 700603) were used as the controls for Kirby/Bauer disk diffusion and combined antibiotic disks method. ${ }^{13}$

\section{Molecular detection of antimicrobial resistance genes and integrons}

Presence of the main genes related to antibiotic resistance, including $\beta$-lactamase encoding, carbapenem resistance, plasmid-mediated quinolone resistance, tetracycline resistance genes, and integrons was investigated using PCR with specific primers for all of the isolates without considering the antibiotic resistance phenotype ${ }^{12,14-20}$ (Table 1). DNA was extracted using the boiling method. ${ }^{21}$ The PCRs were performed at a final volume of $25 \mu \mathrm{L}$ using a green master mix (TSINGKE, China), and the products were visualized by agarose gel $(1 \% \mathrm{w} / \mathrm{v})$ electrophoresis (TSINGKE) stained with ethidium bromide (TSINGKE).

\section{Bacteriophages}

In this study, two specific lytic bacteriophages, vB_SfISISF001 and vB_Ssos-ISF002, against Shigella species were used. ${ }^{22,23}$ For phage proliferation, $500 \mu \mathrm{L}$ phage suspensions (almost $10^{9}$ ) were added to the specific early exponential cultures of the host bacteria (S. sonnei and S. flexneri) and incubated overnight $\left(37^{\circ} \mathrm{C}, 150 \mathrm{rpm}\right)$. To remove bacterial debris, the phage suspensions were centrifuged at $1,000 \mathrm{~g}$ for $10 \mathrm{~min}$, and then the supernatants were filtered using a $0.22 \mu \mathrm{m}$ syringe filter (JinTeng, China) ${ }^{24}$ Phage titers were determined by the double-layer agar method. ${ }^{25}$

\section{Determination of the lytic activity of the phages against clinical isolates of Shigella}

The infection and lysis capacity of vB_SflS-ISF001, vB_Ssos-ISF002, or the cocktail of the two phages against $S$. flexneri and $S$. sonne $i$ isolates were evaluated using the spot test method. ${ }^{23}$ First, the lawn cultures of each isolate were

Table 2. Occurrence of Antimicrobial Resistance Among the Shigella Isolated

\begin{tabular}{|c|c|c|c|c|c|c|}
\hline \multirow[b]{2}{*}{ Antimicrobial agents } & \multicolumn{2}{|c|}{$\begin{array}{l}\text { Resistant } \\
\mathrm{n}(\%)\end{array}$} & \multicolumn{2}{|c|}{$\begin{array}{c}\text { Intermediate } \\
\mathrm{n}(\%)\end{array}$} & \multicolumn{2}{|c|}{$\begin{array}{c}\text { Sensitive } \\
\mathrm{n}(\%)\end{array}$} \\
\hline & S. sonnei & S. flexneri & S. sonnei & S. flexneri & S. sonnei & S. flexneri \\
\hline \multicolumn{7}{|l|}{$\beta$-Lactams } \\
\hline Ampicillin & $42(95.4)$ & $24(92.3)$ & $0(0)$ & $1(3.8)$ & $2(4.5)$ & $1(3.8)$ \\
\hline Cefuroxime & $26(59)$ & $10(38.4)$ & $6(13.6)$ & $6(23)$ & $12(27.2)$ & $10(38.4)$ \\
\hline Ceftazidime & $23(52.2)$ & $10(38.4)$ & $3(6.8)$ & $1(3.8)$ & $18(40.9)$ & $15(57.6)$ \\
\hline Cefotaxime & $27(61.3)$ & $10(38.4)$ & $0(0)$ & $1(3.8)$ & $17(38.6)$ & $15(57.6)$ \\
\hline Cefepime & $4(9)$ & $3(11.5)$ & $4(9)$ & $1(3.8)$ & $36(81.8)$ & $22(84.6)$ \\
\hline \multicolumn{7}{|c|}{ Quinolone and fluoroquinolones } \\
\hline Nalidixic acid & $31(70.4)$ & $16(61.5)$ & $8(18.1)$ & $5(19.2)$ & $5(11.3)$ & $5(19.2)$ \\
\hline Ciprofloxacin & $23(52.2)$ & $8(30.7)$ & $4(9)$ & $4(15.3)$ & $17(38.6)$ & $14(53.8)$ \\
\hline \multicolumn{7}{|l|}{ Polyketides } \\
\hline Tetracycline & $44(100)$ & $26(100)$ & $0(0)$ & $0(0)$ & $0(0)$ & $0(0)$ \\
\hline \multicolumn{7}{|l|}{ Aminoglycosides } \\
\hline Streptomycin & $44(100)$ & $26(100)$ & $0(0)$ & $0(0)$ & $0(0)$ & $0(0)$ \\
\hline \multicolumn{7}{|l|}{ Phenicols } \\
\hline Chloramphenicol & $19(43.1)$ & $9(34.6)$ & $5(11.3)$ & $2(7.6)$ & $20(45.4)$ & $15(57.6)$ \\
\hline
\end{tabular}


prepared, and $10 \mu \mathrm{L}$ of phage suspension $\left(10^{9} \mathrm{pfu} / \mathrm{mL}\right)$ was dropped on the surface of the culture following overnight incubation at $37^{\circ} \mathrm{C}$. The formation of a clear zone at the spot area was considered as bacterial susceptibility to the phage. In the case of plaque formation on host culture, the isolate was subjected to the efficiency of plating (EOP) test using the double-layer agar method. Briefly, EOP was calculated as the ratio of the phage titer of each isolate to the phage titer obtained from the reference bacteria. ${ }^{26}$ The reference bacteria for phage vB_SflS-ISF001 and phage vB_Ssos-ISF002 were
S. flexneri PTCC 1234 and S. sonnei Sh.s-w1, respectively. The test was done in triplicate and results were reported as EOP mean \pm standard deviation.

\section{Preparation of the phage cocktail}

vB_SflS-ISF001 and vB_Ssos-ISF002 phages with a primary concentration of about $10^{9} \mathrm{pfu} / \mathrm{mL}$ were mixed at $1: 1$ ratio and the antibacterial activity was evaluated against the isolates using the spot test method as described above.
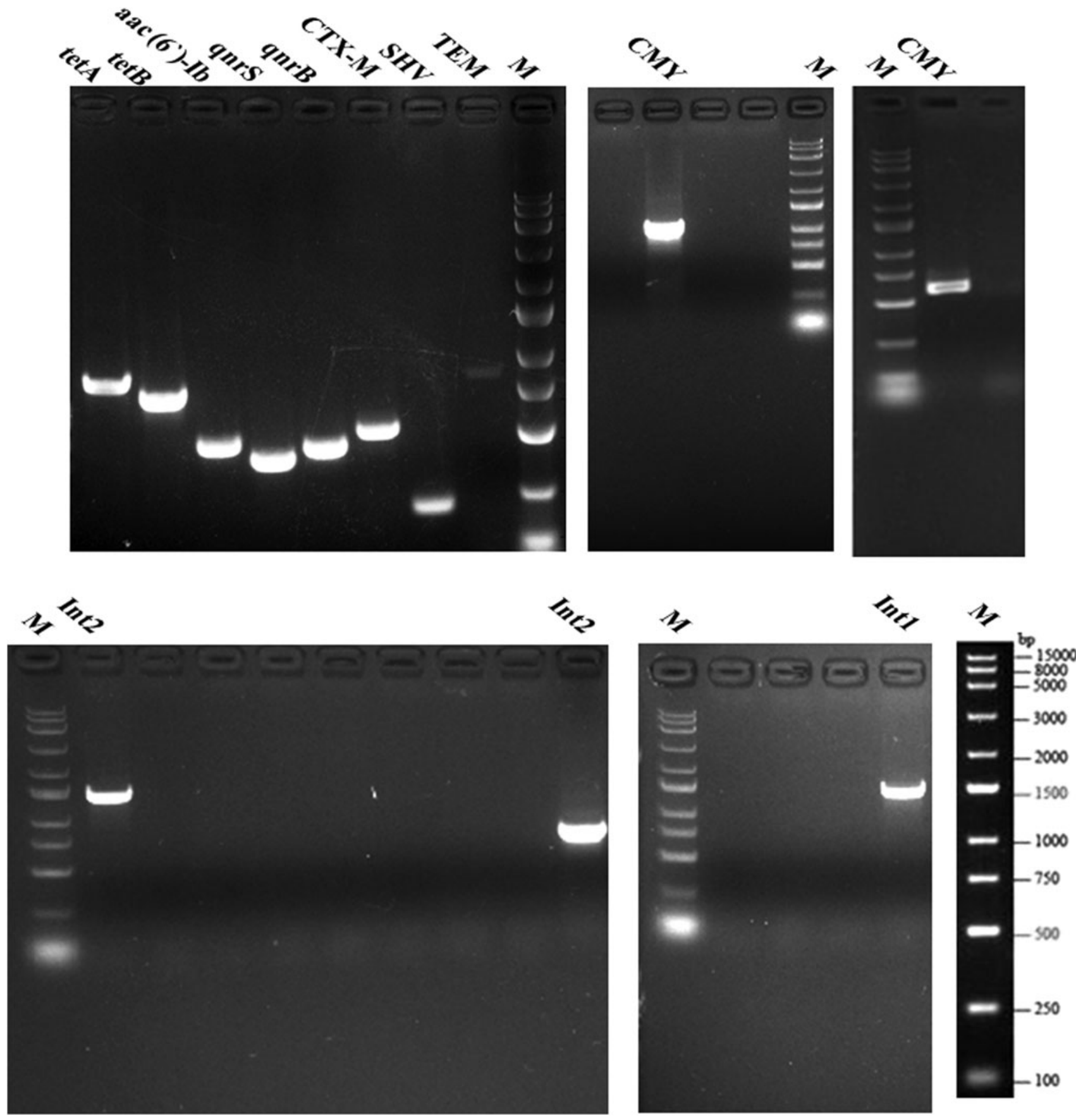

FIG. 1. PCR amplification of the resistance genes and integrons. The gene names are presented at the top of each well. M, DNA marker (NormalRunTM 250bp-IV DNA ladder, ready-to-use) (Generay Biotech Co., Ltd., China). 


\section{Statistical analysis}

The bacteriolytic activities of vB_SflS-ISF001, vB_SsoSISF002, and their cocktail against different $S$. sonnei and $S$. flexneri isolates were analyzed by chi-square test and fisher's exact test using GraphPad Prism software, ver 6.0 (GraphPad Software). $p<0.05$ was considered statistically significant.

\section{Results}

\section{Shigella collection}

Biochemical, molecular, and serogrouping tests were done for all of the obtained clinical isolates of Shigella. The identities of $44 \mathrm{~S}$. sonnei and $26 \mathrm{~S}$. flexneri isolates were confirmed; thus, only these isolates were used for further experiments.

\section{Antimicrobial Susceptibility}

Antimicrobial susceptibility tests showed that all isolates were resistant to tetracycline and streptomycin (Table 2). Without considering these two antibiotics, the lowest $(\sim 10 \%)$ and highest $(\sim 95 \%)$ resistance rates were observed for cefepime and ampicillin, respectively. The antimicrobial susceptibility results also showed that at least $50 \%$ of the $S$. sonnei isolates were resistant against tetracycline, streptomycin, cefotaxime, ceftazidime, cefuroxime, ampicillin, ciprofloxacin, and nalidixic acid. More than $50 \%$ of the $S$. flexneri isolates were resistant against tetracycline, streptomycin, ampicillin, and nalidixic. All the isolates that showed resistance against at least three antibiotic groups were considered as MDR.

\section{Molecular detection of antimicrobial resistance genes and integrons}

Of the 21 tested antimicrobial resistance genes, 12 genes (SHV, TEM, CTX-M, CMY, NDM, qnrB, qnrS, aac, tetA, tet $B$, int 1 , and int2) were found in at least one of the isolates, while $O X A, P E R, V E B, G E S, K P C, I M P$, qnrA, tetC, and tet $D$ genes were not present in any of the isolates (Fig. 1). The frequency of $S H V, T E M, C T X-M, N D M$, qnrS, aac, tetA, and int2 in $S$. sonnei was higher than $S$. flexneri, whereas the frequency of $C M Y$, qnrB, and intl in $S$. flexneri was higher than $S$. sonnei. However, the observed differences were not statistically significant. Excluding the absent genes, the lowest and highest frequencies were observed for $C M Y$ and intl in S. sonnei isolates and NDM and intl in S. flexneri isolates. In addition, the observed frequency of $t e t B$ was the same in both groups (Table 3).

\section{Phenotypic detection of ESBL and minimum inhibitory concentration of imipenem}

The combined antibiotic disks revealed that $59 \%$ of $S$. sonnei (26/44 isolates) and $46.1 \%$ of S. flexneri isolates (12/ 26 isolates) were ESBL producing. Although the number of ESBL-producing isolates in the $S$. sonnei group was higher, the observed difference was not statistically significant ( $p=0.3292)$. PCR results showed that except four isolates of S. sonnei $(9,24,29$, and 30$)$ and nine isolates of $S$. flexneri $(4,8,12,13,16,20,24,25$, and 26), all the other isolates had at least one of the ESBL genes. The lowest and highest MIC of imipenem were 0.094 and $2 \mu \mathrm{g} / \mathrm{mL}$ for both $S$. sonnei and $S$. flexneri isolates (Tables 4 and 5).

\section{Bacteriophage activity against the Shigella isolates}

Spot assay showed that $36.3 \%$ and $72.7 \%$ of S. sonnei isolates were lysed by vB_SflS-ISF001 and vB_SsoSISF002, respectively (Table 6). The observed difference between the sensitivity of $S$. sonnei to the phages was statistically significant. Moreover, vB_SflS-ISF001and vB_SsoSISF002 were capable of producing a clear plaque on $65.3 \%$ and $53.8 \%$ of the $S$. flexneri isolates, respectively, of which the difference was not statistically significant (Table 7).

As shown in Table 6, 11 out of 26 ESBL-producing S. sonnei isolates $(42.3 \%)$ were lysed by vB_SflS-ISF001, and 20 isolates $(76.9 \%)$ were lysed by vB_SsoS-ISF002 $(p=0.0227)$. However, each of the vB_SflS-ISF001 and vB_SsoS-ISF002 bacteriophages lysed 50\% of ESBLproducing $S$. flexneri isolates (6 out of 12 isolates).

EOP results showed that a wide range of phage production varied from $0.03 \pm 0.15$ to $0.02 \pm 0.95$ in different bacterial hosts (Tables 6 and 7). In other words, among 16 S. sonnei isolates susceptible to phage vB_SflS-ISF001, the EOP of 12 isolates was above 0.5 (high production), and for the other 4 isolates, it was under 0.5 (low production). Of the $33 \mathrm{~S}$. sonnei isolates susceptible to phage vB_SsoSISF002, the EOP of 24 isolates was above 0.5 and for the other 9, it was under 0.5 (Table 6). Moreover, among the 17 isolates of $S$. flexneri lysed by phage vB_SflS-ISF001, 11

\section{Table 3. Occurrence of Different Antimicrobial Resistance Genes and Integrons Among THE SHIGELLA ISOLATES}

\begin{tabular}{lcc}
\hline Antibiotic resistance genes & $\begin{array}{c}\text { S. sonnei } \\
\mathrm{n}(\%)\end{array}$ & $\begin{array}{c}\text { S. flexneri } \\
\mathrm{n}(\%)\end{array}$ \\
\hline Extended-spectrum $\beta$-lactamases & & \\
SHV & $7(15.9)$ & $3(11.5)$ \\
TEM & $25(56.8)$ & $14(53.8)$ \\
CTX-M & $21(47.7)$ & $7(26.9)$ \\
OXA & $0(0)$ & $0(0)$ \\
PER & $0(0)$ & $0(0)$ \\
VEB & $0(0)$ & $0(0)$ \\
GES & $0(0)$ & $0(0)$ \\
CMY & $2(4.5)$ & $2(7.6)$ \\
KPC & $0(0)$ & $0(0)$ \\
NDM & $3(6.8)$ & $1(3.8)$ \\
IMP & $0(0)$ & $0(0)$ \\
Quinolone resistance & & \\
qnrA & $0(0)$ & $0(0)$ \\
qnrB & $10(22.7)$ & $7(26.9)$ \\
qnrS & $14(31.8)$ & $4(15.3)$ \\
Aac & $17(38.6)$ & $9(34.6)$ \\
Tetracycline resistance & & \\
tetA & $19(43.1)$ & $9(34.6)$ \\
tetB & $22(50)$ & $13(50)$ \\
tetC & $0(0)$ & $0(0)$ \\
tetD & $0(0)$ & $0(0)$ \\
Integron & & \\
int1 & $28(63.6)$ & $17(65.3)$ \\
int2 & $17(38.6)$ & $10(38.4)$ \\
\hline
\end{tabular}


Table 4. Resistance Phenotypes and Resistance Genes in Clinical Isolates of $S$. sonneI

\begin{tabular}{|c|c|c|c|c|}
\hline $\begin{array}{l}\text { S. sonnei } \\
\text { isolates }\end{array}$ & Resistance phenotypes & $E S B L$ & $\begin{array}{l}\text { MIC of IMI } \\
\quad(\mu g / m L)\end{array}$ & Resistance genes/integron \\
\hline $\mathrm{C} 1$ & TET, STR, CHL, AMP, NAL, CIP, CTX, FEP & + & 0.19 & $C T X-M, q n r B, a a c$, tetB, intl \\
\hline $\mathrm{C} 2$ & TET, STR, AMP, NAL, CIP, CXM, CAZ, CTX, FEP & + & 0.125 & TEM, CTX-M, qnrS, tetA, int1 \\
\hline $\mathrm{C} 3$ & TET, STR, CHL, AMP, NAL, CIP, CXM, CAZ, CTX & + & 0.125 & $C T X-M, q n r S$, tet $B$, int 1 \\
\hline $\mathrm{C} 4$ & TET, STR, AMP, NAL, CXM, CTX & + & 0.094 & $T E M, S H V, q n r B$, tetB, int 2 \\
\hline $\mathrm{C} 5$ & TET, STR, CHL, AMP & - & 0.5 & $T E M$, int 1 \\
\hline C6 & TET, STR, AMP & - & 0.125 & TEM, int1 \\
\hline $\mathrm{C} 7$ & TET, STR, CHL, AMP, NAL, CIP, CXM, CAZ, CTX & + & 0.125 & $C M Y, q n r B$, tetB, int 2 \\
\hline $\mathrm{C} 8$ & TET, STR, AMP, NAL, CIP, CXM, CAZ, CTX, FEP & + & 2 & $C T X-M, N D M, q n r B$, tetB, int 2 \\
\hline C9 & TET, STR, AMP, NAL, CIP & - & 0.125 & qnrS, tetA, int1 \\
\hline $\mathrm{C} 10$ & TET, STR, CHL, AMP, NAL & - & 0.125 & $T E M, C T X-M, a a c$, tet $B$, int 2 \\
\hline $\mathrm{C} 11$ & TET, STR, AMP, NAL, CXM, CAZ, CTX & + & 1 & $T E M, N D M, a a c$, tetA, int 1 \\
\hline $\mathrm{C} 12$ & TET, STR, CHL, AMP, NAL, CIP, CXM, CAZ, CTX & + & 0.75 & $C T X-M, q n r S, a a c$, tet $B$, int 1 \\
\hline $\mathrm{C} 13$ & TET, STR, AMP, NAL, CXM, CAZ, CTX, FEP & + & 0.125 & $T E M, S H V, q n r S$, tetA, int 2 \\
\hline $\mathrm{C} 14$ & TET, STR, CHL, AMP, NAL, CAZ, CTX & + & 0.125 & $C T X-M, a a c$, tet $B$, int 1 \\
\hline $\mathrm{C} 15$ & TET, STR, AMP, CXM, CAZ, CTX & + & 0.5 & $T E M, C T X-M$, tetA, int 1 \\
\hline $\mathrm{C} 16$ & TET, STR, CHL, AMP, NAL, CIP, CXM, CTX & + & 0.125 & $C T X-M$, qnrS, tet $B$, int 2 \\
\hline $\mathrm{C} 17$ & TET, STR, AMP, CIP & - & 0.125 & $T E M$, aac, tetB, int 2 \\
\hline $\mathrm{C} 18$ & TET, STR, AMP, NAL, CIP, CXM, CAZ, CTX & + & 0.125 & $S H V, C T X-M, q n r B$, intl \\
\hline C19 & TET, STR, AMP, CXM, CAZ, CTX & + & 0.125 & $S H V, C T X-M, a a c$, tet $B$, int 1 \\
\hline $\mathrm{C} 20$ & TET, STR, AMP, NAL & - & 0.125 & $T E M$, aac, tetA, int 2 \\
\hline $\mathrm{C} 21$ & TET, STR, AMP, CXM, CAZ, CTX & + & 0.5 & $T E M, C T X-M, a a c$, tet $B$, int 1 \\
\hline $\mathrm{C} 22$ & TET, STR, CHL, NAL, CIP, CXM, CAZ, CTX & + & 0.125 & $C T X-M, q n r S$, tetA, int 2 \\
\hline $\mathrm{C} 23$ & TET, STR, CHL, AMP, NAL, CIP, CXM, CAZ, CTX & + & 0.125 & $T E M, C T X-M, q n r S$, tetA, int 2 \\
\hline $\mathrm{C} 24$ & TET, STR, NAL & - & 0.125 & aac, intl \\
\hline $\mathrm{C} 25$ & TET, STR, AMP & - & 0.125 & $T E M$, tet $A$, tet $B$, int 1 \\
\hline $\mathrm{C} 26$ & TET, STR, AMP, CXM & - & 0.125 & $S H V$, int 2 \\
\hline $\mathrm{C} 27$ & TET, STR, AMP, NAL, CIP, CXM, CAZ, CTX & - & 0.125 & $\begin{array}{l}T E M, S H V, C T X-M, q n r B, q n r S \text {, } \\
\text { tetB, int1 }\end{array}$ \\
\hline $\mathrm{C} 28$ & TET, STR, CHL, NAL, CIP, CXM, CAZ & - & 0.125 & $T E M, q n r S$, tet $A$, int 1 \\
\hline $\mathrm{C} 29$ & TET, STR, NAL & - & 0.125 & tetA, int 2 \\
\hline $\mathrm{C} 30$ & TET, STR, CHL, AMP, NAL, CXM, CTX & + & 0.19 & aac, tet $B$, int 1 \\
\hline $\mathrm{C} 31$ & TET, STR, AMP, CXM, CAZ, CTX & + & 0.125 & $S H V, C T X-M, a a c$, int 1 \\
\hline C32 & TET, STR, CHL, AMP & - & 0.125 & $T E M$, aac, tet $B$, int 1 \\
\hline $\mathrm{C} 33$ & TET, STR, CHL, AMP, NAL, CIP & - & 0.094 & $T E M, q n r B$, tet $A$, int 2 \\
\hline $\mathrm{C} 34$ & TET, STR, AMP, NAL, CIP & - & 0.75 & $T E M$, qnrS, tet $A$, int 2 \\
\hline $\mathrm{C} 35$ & TET, STR, AMP & - & 0.125 & $T E M$, tet $B$, int 2 \\
\hline $\mathrm{C} 36$ & TET, STR, AMP, NAL, CIP, CTX & + & 0.19 & $T E M, q n r S$, tetA, tetB, int 2 \\
\hline C37 & TET, STR, AMP,CHL, NAL, CIP, CXM, CAZ, CTX & + & 0.125 & $T E M, C T X-M$, qnrS, tetA, int 1 \\
\hline $\mathrm{C} 38$ & TET, STR, AMP, NAL, CIP & - & 0.125 & $T E M$, aac, tet $A$, tet $B$, int 1 \\
\hline C39 & TET, STR, CHL, AMP, NAL, CIP, CXM, CAZ, CTX & + & 0.094 & $T E M, C M Y, q n r S$, tetB, intl \\
\hline $\mathrm{C} 40$ & TET, STR, CHL, AMP, NAL, CIP, CXM, CAZ, CTX & + & 0.75 & $\begin{array}{l}\text { TEM, } C T X-M, q n r B, a a c, \\
\text { tetB, int } 2\end{array}$ \\
\hline $\mathrm{C} 41$ & TET, STR, CHL, AMP, NAL, CIP, CXM, CAZ, CTX & + & 0.125 & $C T X-M$, qnrB, tetA, int1 \\
\hline $\mathrm{C} 42$ & TET, STR, AMP & - & 0.125 & $T E M$, aac, tetA, int 1 \\
\hline $\mathrm{C} 43$ & TET, STR, CHL, AMP, NAL, CIP, CXM, CAZ, CTX & + & 1.5 & $C T X-M, N D M, q n r B$, tetB, intl \\
\hline $\mathrm{C} 44$ & TET, STR, AMP, NAL, CXM, CAZ, CTX & + & 0.125 & $C T X-M$, aac, tetA, int 1 \\
\hline
\end{tabular}

AMK, amikacin; AMP, ampicillin; CAZ, ceftazidime; CHL, chloramphenicol; CIP, ciprofloxacin; CTX, cefotaxime; CXM, cefuroxime; ESBL, extended-spectrum $\beta$-lactamase; -, ESBL negative; +, ESBL positive; FEP, cefepime; IMI, imipenem; MIC, minimum inhibitory concentration; NAL, nalidixic acid; STR, streptomycin; TET, tetracycline.

and 6 isolates had an EOP of above and under 0.5, respectively; whereas, for the $14 \mathrm{~S}$. flexneri isolates susceptible to phage vB_SsoS-ISF002, the EOP of 10 and 4 isolates were above and under 0.5 , respectively (Table 7 ).

\section{Phage cocktail}

Among all of the $44 S$. sonnei and 26 S. flexneri isolates, only 5 isolates were not found sensitive to the phage cocktail. In other words, $88.6 \%$ of the $S$. sonnei isolates and
$80.7 \%$ of $S$. flexneri isolates were lysed by the cocktail. In ESBL-producing isolates, $88.4 \%$ of the $S$. sonnei (23 out of 26 ), and $75 \%$ of the $S$. flexneri isolates (9 out of 12) were lysed by the cocktail.

\section{Discussion}

As one of the most important pathogens associated with diarrhea, Shigella is a global health concern in particular in children under the age of 5 years. ${ }^{27}$ Shigellosis is generally 
Table 5. Resistance Phenotypes and Resistance Genes in Clinical Isolates of S. FlexNeri

\begin{tabular}{|c|c|c|c|c|}
\hline $\begin{array}{l}\text { S. flexneri } \\
\text { isolates }\end{array}$ & Phenotypic resistance profile & $E S B L$ & $\begin{array}{l}\text { MIC of IMI } \\
\quad(\mu \mathrm{g} / \mathrm{mL})\end{array}$ & Resistance genes/integron \\
\hline $\mathrm{C} 1$ & TET, STR, TET, STR, CHL, NAL, CXM, CTX & + & 0.5 & $T E M, C M Y, q n r B$, tet $A$, int 1 \\
\hline $\mathrm{C} 2$ & TET, STR, AMP & - & 0.125 & $T E M, a a c$, tetB, intl \\
\hline $\mathrm{C} 3$ & TET, STR, AMP, CAZ & + & 0.125 & $S H V, C T X-M, a a c$, tet $B$, int 2 \\
\hline $\mathrm{C} 4$ & TET, STR, AMP & - & 0.125 & qnrS, int1 \\
\hline $\mathrm{C} 5$ & TET, STR, AMP, CXM, CTX & + & 0.125 & TEM, $C T X-M$, tet $A$, int 1 \\
\hline C6 & TET, STR, NAL & - & 0.094 & $T E M, q n r S$, tetA, int 2 \\
\hline $\mathrm{C} 7$ & TET, STR, AMP, NAL, CIP, CXM, CAZ, CTX & + & 0.19 & $C M Y, q n r B$, tetB, int 1 \\
\hline $\mathrm{C} 8$ & TET, STR, CHL, AMP & - & 0.125 & int 1 \\
\hline C9 & TET, STR, AMP, CAZ & + & 0.125 & $T E M, C T X-M$, tetA, int 1 \\
\hline $\mathrm{C} 10$ & TET, STR, AMP & - & 0.094 & $T E M$, tetA, int 2 \\
\hline $\mathrm{C} 11$ & TET, STR, AMP, NAL, CIP, CXM, CAZ, CTX, FEP & + & 2 & $\begin{array}{l}T E M, C T X-M, N D M, q n r B, \\
\text { aac, tet } A, \text { tet } B, \text { int1 }\end{array}$ \\
\hline $\mathrm{C} 12$ & TET, STR, CHL, AMP, NAL, CIP & - & 0.125 & $a a c$, tet $B$, int 1 \\
\hline C13 & TET, STR, AMP & - & 0.125 & tet $B$, int 2 \\
\hline C14 & TET, STR, AMP, NAL, CXM, CAZ, CTX & + & 0.125 & $T E M, S H V, q n r S$, tetA, intl \\
\hline C15 & TET, STR, AMP, NAL, CIP, CXM, CAZ, CTX, FEP & + & 0.5 & $T E M, q n r B$, int 1 \\
\hline C16 & TET, STR, AMP, NAL & - & 0.125 & $a a c$, tetB, int 2 \\
\hline $\mathrm{C} 17$ & TET, STR, AMP, NAL, CIP, CXM, CAZ, CTX, FEP & + & 0.38 & $T E M, C T X-M$, aac, tetA, int 1 \\
\hline C18 & TET, STR, AMP, NAL, CXM, CAZ, CTX & - & 0.125 & $T E M$, aac, tetB, int 2 \\
\hline C19 & TET, STR, CHL, AMP, NAL, CIP & - & 0.125 & $T E M$, qnrB, tetB, int 2 \\
\hline $\mathrm{C} 20$ & TET, STR, CHL, AMP & - & 0.094 & tet $B$, int1 \\
\hline $\mathrm{C} 21$ & TET, STR, CHL, AMP, NAL, CXM, CTX & + & 0.19 & $C T X-M, q n r S$, tet $B$, int 1 \\
\hline $\mathrm{C} 22$ & TET, STR, CHL, AMP, NAL, CIP, CXM, CAZ, CTX & + & 0.19 & $T E M, S H V, a a c$, tetB, int 2 \\
\hline $\mathrm{C} 23$ & TET, STR, AMP, NAL, CAZ & + & 0.125 & $T E M, C T X-M, q n r B$, tet $A$, int 2 \\
\hline $\mathrm{C} 24$ & TET, STR, AMP & - & 0.094 & tetB, int1 \\
\hline $\mathrm{C} 25$ & TET, STR, CHL, AMP, NAL & - & 0.094 & aac, int 2 \\
\hline $\mathrm{C} 26$ & TET, STR, CHL, AMP, NAL, CIP & - & 0.094 & $q n r B$, intl \\
\hline
\end{tabular}

introduced as the disease for poor, crowded populations who lack adequate public or personal hygiene, clean water, and proper wastewater sanitation systems. This pathogen has become resistant to several classes of antibiotics, such as cephalosporins and quinolones, which further burden the clinical procedures to identify effective antibiotics against this microorganism, ${ }^{6}$ of which the ESBL-producing strains are of critical importance. ${ }^{6}$ Therefore, there is an urgent need in finding and developing new strategies to control or eliminate the MDR and/or ESBL-producing isolates of Shigella. Using lytic bacteriophages against bacterial pathogens (phage therapy) is one of the promising alternatives in which its effectiveness is independent of the strain being MDR or not, or being an ESBL positive or an ESBL negative. ${ }^{28}$

The objectives of our study were to determine the presence of any of the 21 antimicrobial resistance genes and integrons among the clinical MDR isolates of Shigella, identification of the ESBL-producing isolates, and finally, studying the efficiency of vB_SflS-ISF001, vB_SsoS-ISF002, and their cocktail on the ESBL-positive and -negative isolates.

In a study by Nuesch et al. during 2004-2014 on 344 Shigella spp isolates, $78.5 \%$ of the isolates were identified as MDR. In detail, $83.8 \%$ of S. sonnei and $78.5 \%$ of $S$. flexneri isolates were MDR. Moreover, the resistant levels to ampicillin, cefotaxime, nalidixic acid, streptomycin, tetracycline, ciprofloxacin, and chloramphenicol were $17.3 \%$, $4.5 \%, 27.4 \%, 91 \%, 81 \%, 15 \%$, and $3.4 \%$ in the $S$. sonnei isolates and $68.2 \%, 0 \%, 14 \%, 75.7 \%, 77.6 \%, 8.4 \%$, and $52.3 \%$ in the $S$. flexneri isolates, respectively. ${ }^{29}$ The study by Ghosh et al. during 2007-2010 reported 90\% of the
Shigella isolates as MDR, and the resistance measures to nalidixic acid, tetracycline, ciprofloxacin, chloramphenicol, and ampicillin in these isolates were recorded as $93.4 \%, 88.7 \%, 85.8 \%, 62.7 \%$, and $53.8 \%$, respectively. ${ }^{30}$ The emergence of antibiotic-resistant pathogens, such as Shigella species, has become a global concern. ${ }^{2}$ Comparing the antimicrobial susceptibility obtained in this study with the previous reports disclose a catastrophical increase in the frequency of antibiotic-resistant Shigella species over the past few years. Williams and Berkley reported that resistance to the third generation of the cephalosporins due to increased number of the ESBL-producing bacteria has been increasingly prevalent in Asia, and suggested monitoring plans for such strains. ${ }^{31}$ The relatively high rate of ESBL isolates in this study $(59 \%$ and $46.1 \%$ for $S$. sonnei and $S$. flexneri, respectively) indicates a significant increase in the population of these superbugs and calls for urgent actions in developing new efficient alternatives for rapid detection, monitoring, and control/treatment strategies.

Horizontal transmission of antibiotic resistance genes between close species or even genera of bacteria can convert susceptible isolates to resistant ones, thus, it is an important cause of the progressive increase in the frequency of the resistant isolates. Mandomando et al. reported frequency of $71 \%$ and $2 \%$ for the tetA in S. sonnei and S. flexneri, respectively, whereas it was $0 \%$ and $98 \%$ for $t e t B$, respectively. ${ }^{32}$ Beladi et al. reported the frequencies of TEM, CTX-M, and $S H V$ genes in $S$. sonnei isolates as $60.87 \%, 21.74 \%$, and $0 \%$, and $58.3 \%, 16.6 \%$, and $0 \%$ in $S$. flexneri isolates, respectively. ${ }^{33}$ Hartman et al. study on 459 tetracyclineresistant Shigella isolates reported the frequency of tetA and 


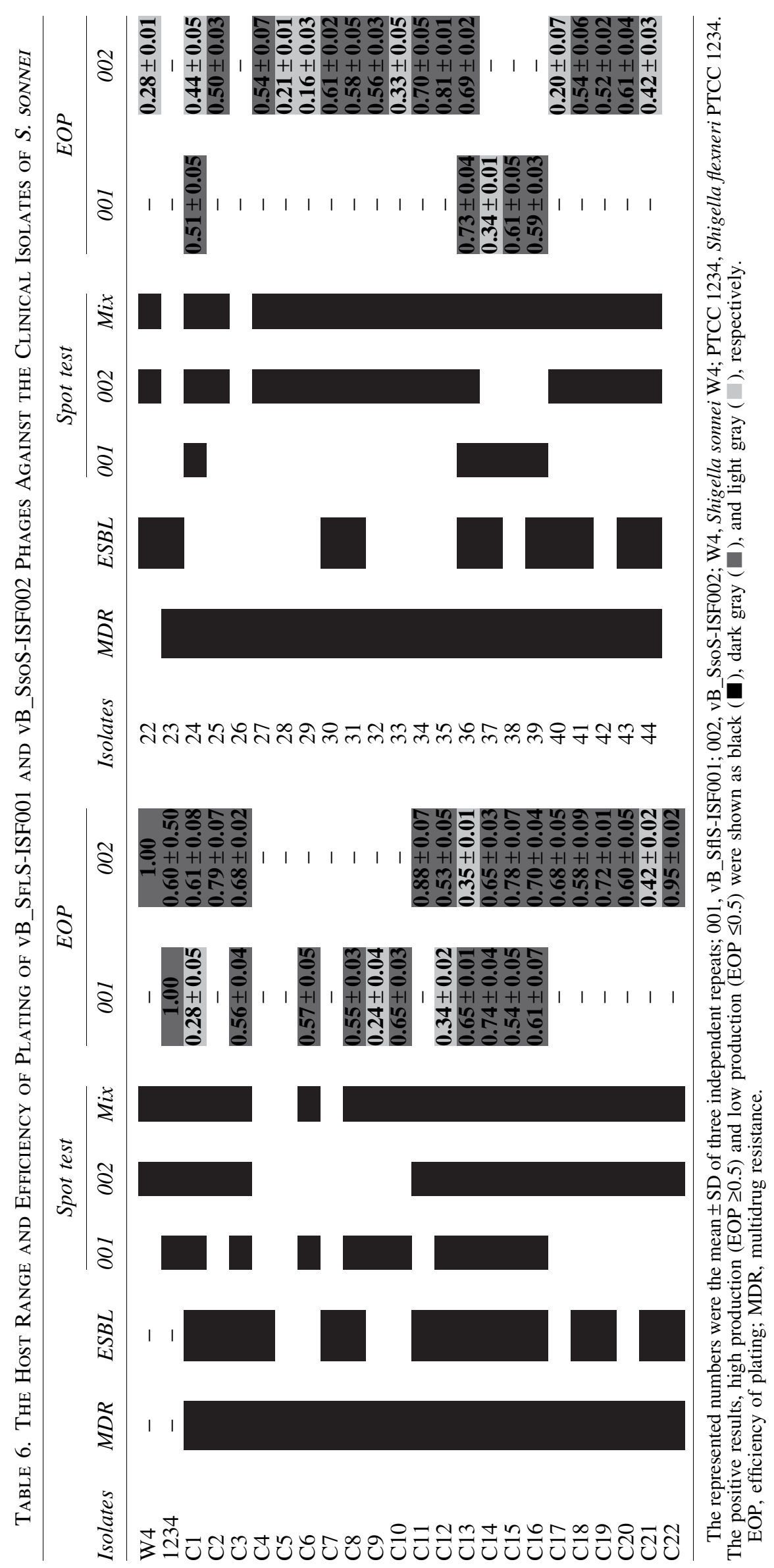


Table 7. The Host Range and Efficiency of Plating of vB_SflS-ISF001 and vB_SsoS-ISF002 Phages Against the Clinical Isolates of $S$. FLEXNERI

\begin{tabular}{|c|c|c|c|c|c|c|c|}
\hline \multirow[b]{2}{*}{ Isolates } & \multirow[b]{2}{*}{$M D R$} & \multirow[b]{2}{*}{$E S B L$} & \multicolumn{3}{|c|}{ Spot test } & \multicolumn{2}{|c|}{$E O P$} \\
\hline & & & 001 & 002 & Mix & 001 & 002 \\
\hline W4 & - & - & - & & & - & 1.00 \\
\hline PTCC 1234 & - & - & & & & 1.00 & $0.60 \pm 0.50$ \\
\hline $\mathrm{C} 1$ & & & & & & $0.53 \pm 0.02$ & $0.37 \pm 0.05$ \\
\hline $\mathrm{C} 2$ & & & & & & $0.15 \pm 0.03$ & $0.65 \pm 0.06$ \\
\hline $\mathrm{C} 3$ & & & & & & $0.40 \pm 0.05$ & $0.67 \pm 0.01$ \\
\hline $\mathrm{C} 4$ & & & & & & $0.72 \pm 0.02$ & $0.62 \pm 0.03$ \\
\hline C5 & & & & & & - & $0.80 \pm 0.05$ \\
\hline C6 & & & & & & $0.52 \pm 0.05$ & $0.44 \pm 0.02$ \\
\hline C7 & & & & & & - & $0.68 \pm 0.06$ \\
\hline $\mathrm{C} 8$ & & & & & & $0.37 \pm 0.05$ & $0.29 \pm 0.04$ \\
\hline C9 & & & & & & - & $0.75 \pm 0.01$ \\
\hline $\mathrm{C} 10$ & & & & & & - & - \\
\hline $\mathrm{C} 11$ & & & & & & - & - \\
\hline $\mathrm{C} 12$ & & & & & & $0.65 \pm 0.02$ & $0.53 \pm 0.05$ \\
\hline $\mathrm{C} 13$ & & & & & & $0.59 \pm 0.03$ & $0.67 \pm 0.01$ \\
\hline $\mathrm{C} 14$ & & & & & & - & - \\
\hline $\mathrm{C} 15$ & & & & & & - & - \\
\hline $\mathrm{C} 16$ & & & & & & - & - \\
\hline $\mathrm{C} 17$ & & & & & & $0.50 \pm 0.07$ & $0.15 \pm 0.02$ \\
\hline $\mathrm{C} 18$ & & & & & & $0.62 \pm 0.01$ & $0.73 \pm 0.05$ \\
\hline C19 & & & & & & - & $0.87 \pm 0.02$ \\
\hline $\mathrm{C} 20$ & & & & & & $0.42 \pm 0.01$ & - \\
\hline $\mathrm{C} 21$ & & & & & & $0.73 \pm 0.06$ & - \\
\hline $\mathrm{C} 22$ & & & & & & $0.81 \pm 0.01$ & - \\
\hline $\mathrm{C} 23$ & & & & & & $0.55 \pm 0.05$ & - \\
\hline $\mathrm{C} 24$ & & & & & & $0.27 \pm 0.08$ & - \\
\hline $\mathrm{C} 25$ & & & & & & $0.45 \pm 0.01$ & - \\
\hline $\mathrm{C} 26$ & & & & & & $0.64 \pm 0.03$ & - \\
\hline
\end{tabular}

The represented numbers are the mean \pm SD of three independent repeats; W4, Shigella sonnei $\mathrm{W} 4$; PTCC 1234, Shigella flexneri PTCC 1234. The positive results, high production (EOP $\geq 0.5$ ) and low production (EOP $\leq 0.5)$ were shown as black $(\boldsymbol{\square})$, dark gray $(\boldsymbol{\square})$ and light gray $(\square)$, respectively.

tetB genes as $26 \%$ and $75 \%$ for the S. flexneri isolates, whereas no tet $C$ and $t e t B$ genes were detected. The frequencies of tetA, tetB, tet $C$, and tetD genes in $S$. sonnei isolates were recorded as $16 \%, 36 \%, 3 \%$, and $42 \%$, respectively. ${ }^{34}$ Sow et al. reported the frequencies of class 1 and class 2 integrons in Shigella spp. as $92.8 \%$ and $57.1 \%$, respectively. ${ }^{35}$ Barrantes and Achí review on Shigella spp. found a significant geographical difference in the prevalence of class 1 and class 2 integrons in Brazil, Bolivia, Chile, Costa Rica, and Peru. ${ }^{36}$ The observed differences among these studies can be due to geographical differences (e.g., different antibiotic consumption pattern in different countries), the number of samples, human errors, and the type of materials in experiments and sampling; nevertheless, the overall global pattern indicates an increasing trend in the spread of antibiotic resistance genes. Enterobacteriaceae family members, as one of the most clinically significant microorganisms, are capable of horizontal gene transmission among the family members and contain many of these resistance genes in their genome. ${ }^{37}$ Most of these genes have extrachromosomal DNA origins, and exposure to the related antibiotics, activates the expression of these genes; while, in the absence of such environmental pressures, bacteria lose these plasmids due to the fitness cost. Therefore, in theory, the use of alternative methods can lead to a significant decrease in antibiotic con- sumption, and over time may decrease the antibiotic-resistant incidence, which, as a result, may prevent the epidemics of antibiotic-resistant infectious diseases. ${ }^{38-40}$

As early as the discovery of the bacteriophage in the 1910s, their potential use in treatment of bacterial infections, also known as phage therapy, was recognized. ${ }^{27}$ Nowadays, phage therapy is one of the most promising alternative methods for antibiotic therapy. The remarkable properties of phages, including having specific hosts and as a result having no harmful influences on the normal flora or natural microbial communities of an ecosystem, productive potential, and having no side effects on eukaryotic cells, make them a highly efficient and suitable alternative for antibiotic agents. ${ }^{7,9}$ Several phage-based antibacterial products, such as Salmofresh, Ecolshield, Listshield, and Phaeburn that are already approved by the FDA and are available in the trade market, demonstrate the confirmation of successful application.

The application of bacteriophages in phage cocktail(s) requires specific criteria. An essential criterion for the application of bacteriophages in phage therapy or biocontrol purposes is being safe to use. The appropriate potential of vB_SfIS-ISF001 to be used as a biocontrolling agent in raw or cooked food had been described previously. ${ }^{22}$ In addition, no undesirable or harmful genes, example, antimicrobial resistance, bacterial virulence, or lysogeny genes were found 
in the whole genome sequences of vB_SfIS-ISF001 (GenBank accession number: MG049919) and vB_SsoS-ISF002 (GenBank accession number: MF093736). ${ }^{23,4 \overline{1}}$

Having a specific phage/bacteria interaction and yet having the capacity to lyse different pathogenic strains/species of the same bacterial genus is another important criterion for the efficient application of bacteriophages. We demonstrated that both vB_SflS-ISF001 and vB_SsoS-ISF002 were lytic bacteriophages against most of the $S$. sonnei and $S$. flexneri isolates, that is, both phages have a wide host range. Nevertheless, not being able to lyse a few of the isolates of both species does not affect such capacity. Adding one or more specific and safe bacteriophage(s) to the cocktail is a solution to overcome this shortage.

Being active against either antibiotic-resistant or susceptible strains is another critical feature for an effective bacteriophage product. vB_SfIS-ISF001 and vB_SsoS-ISF002 ability to lyse ESBL-producing MDR isolates of both $S$. sonnei and S. flexneri isolates is an indication of a high potential of these phages to be used as a phage product, in particular, a phage cocktail. ShigActive ${ }^{\mathrm{TM}}$, a phage cocktail consisting of five lytic Shigella bacteriophages, could inhibit the growth of about $92 \%$ of $S$. flexneri strains. ${ }^{42}$ Bernasconi et al. evaluated three commercial phage cocktails (PYO, INTESTI, Septaphage) against Shigella spp., and reported 95\% susceptibility (19 out of 20 isolates) for the tested Shigella isolates to PYO and INTESTI, and $55 \%$ susceptibility (11 out of 20 isolates) to Septaphage. ${ }^{43}$ However, none of these studies focused on the effects of these phage cocktails on MDR and ESBL-producing isolates. Also, we observed a high EOP for many of the bacterial isolates, which indicate desirable phage production in most of the samples and can ensure effective production and a high number of newborn phage release. The results of our study demonstrate that vB_SfIS-ISF001 and vB_SsoS-ISF002 are highly potent means of elimination for Shigella contaminations. Thus, the high efficiency of elimination against the two important Shigella species hosts by these phages is an indication of their potential for further development and commercial use of the phage cocktail against Shigella.

In summary, antibiotic resistance and high prevalence of ESBL-producing Shigella and the resistance genes should be considered as a serious public health issue. With the increasing number of antibiotic-resistant bacteria, bacteriophages are a powerful option for the postantibiotic era. Using vB_SflS-ISF001 and vB_SsoS-ISF002 bacteriophages or a cocktail of both phages have the potential of further development to an antimicrobial agent against Shigella superbugs despite the MDR and/or ESBL profile.

\section{Acknowledgment}

The authors would like to thank all colleagues who made this research successful.

\section{Disclosure Statement}

No competing financial interests exist.

\section{Funding Information}

This work was supported by the National Natural Science Foundation of China (31950410562 and 31702297) and Natural Science Foundation of Jiangsu Province (BK20160577). The authors would like to thank the University of Isfahan for financial support (A/94/32650).

\section{References}

1. Shahin, K., M. Bouzari, R. Wang, and M.R. Khorasgani. 2019. Distribution of antimicrobial resistance genes and integrons among Shigella spp. isolated from water sources. J. Glob. Antimicrob. Res. 19:122-128.

2. Puzari, M., M. Sharma, and P. Chetia. 2018. Emergence of antibiotic resistant Shigella species: a matter of concern. J. Infect. Public Health 11:451-454.

3. Mokomane, M., I. Kasvosve, E. de Melo, J.M. Pernica, and D.M. Goldfarb. 2018. The global problem of childhood diarrhoeal diseases: emerging strategies in prevention and management. Ther. Adv. Infect. Dis. 5:29-43.

4. Ventola, C.L. 2015. The antibiotic resistance crisis: part 1: causes and threats. P. T. 40:277-283.

5. CDC. 2019. National Antimicrobial Resistance Monitoring System (NARMS) Now: Human Data. U.S. Department of Health and Human Services, CDC, Atlanta, GA (https://cdc .gov/narmsnow).

6. WHO. 2017. WHO Publishes List of Bacteria for which New Antibiotics are Urgently Needed. World Health Organization, Geneva, Switzerland.

7. Lin, D.M., B. Koskella, and H.C. Lin. 2017. Phage therapy: an alternative to antibiotics in the age of multi-drug resistance. World J. Gastrointest. Pharmacol. Ther. 8:162-173.

8. Shahin, K., H. Bao, M. Komijani, M. Barazandeh, M. Bouzari, A. Hedayatkhah, L. Zhang, H. Zhao, T. He, M. Pang, and R. Wang. 2019. Isolation, characterization, and PCR-based molecular identification of a siphoviridae phage infecting Shigella dysenteriae. Microb. Pathog. 131:175180.

9. Rezaei, M., M. Komijani, and S.M. Javadirad. 2012. Bacteriostatic Agents. A Search for Antibacterial Agents. IntechOpen, London.

10. Ahmed, A.M., and T. Shimamoto. 2014. Isolation and molecular characterization of Salmonella enterica, Escherichia coli O157: H7 and Shigella spp. from meat and dairy products in Egypt. Int. J. Food Microbiol. 168:57-62.

11. Mikoleit, M. 2010. Laboratory Protocol: Biochemical Identification of Salmonella and Shigella using an Abbreviated Panel of Tests. WHO Global Foodborne Infections Network, Geneva, Switzerland.

12. Alizadeh-Hesar, M., B. Bakhshi, and S. Najar-Peerayeh. 2015. Clonal dissemination of a single Shigella sonnei strain among Iranian children during fall 2012 in Tehran, IR Iran. Infect. Genet. Evol. 34:260-266.

13. CLSI. 2014. Performance Standards for Antimicrobial Susceptibility Testing; Twenty-fourth Informational Supplement. CLSI, Wayne, PA.

14. Ahmed, A.M., and T. Shimamoto. 2015. Molecular characterization of multidrug-resistant Shigella spp. of food origin. Int. J. Food Microbiol. 194:78-82.

15. Ahmed, A.M., and T. Shimamoto. 2015. Molecular analysis of multidrug resistance in Shiga toxin-producing Escherichia coli O157: $\mathrm{H} 7$ isolated from meat and dairy products. Int. J. Food Microbiol. 193:68-73.

16. Kim, J., S.G. Hong, I.K. Bae, J.R. Kang, S.H. Jeong, W. Lee, and K. Lee. 2011. Emergence of Escherichia coli sequence type ST131 carrying both the blaGES-5 and blaCTX-M-15 genes. Antimicrob. Agents Cahemother. 55:2974-2975.

17. Komijani, M., M. Bouzari, and F. Rahimi. 2017. Detection and characterization of a novel lytic bacteriophage (vBKpneM-Isf48) against Klebsiella pneumoniae isolates from infected wounds carrying antibiotic-resistance genes (TEM, SHV, and CTX-M). Iran. Red Crescent Med. J. 19. 
18. Neyestanaki, D.K., A. Mirsalehian, F. Rezagholizadeh, F. Jabalameli, M. Taherikalani, and M. Emaneini. 2014. Determination of extended spectrum beta-lactamases, metallo-beta-lactamases and AmpC-beta-lactamases among carbapenem resistant $P$ seudomonas aeruginosa isolated from burn patients. Burns 40:1556-1561.

19. Nordmann, P., T. Naas, and L. Poirel. 2011. Global spread of carbapenemase-producing Enterobacteriaceae. Emerg. Infect. Dis. 17:1791-1798.

20. Peerayeh, S.N., R.P. Mahabadi, S.P. Toupkanlou, and S.D. Siadat. 2014. Diversity of $\beta$-lactamases produced by imipenem resistant, Pseudomonas aeruginosa isolates from the bloodstream. Burns 40:1360-1364.

21. Abdelhai, M.H., H. Hassanin, and X. Sun. 2016. Comparative study of rapid DNA extraction methods of pathogenic bacteria. Am. J. Biosci. Bioeng. 4:1-8.

22. Shahin, K., and M. Bouzari. 2018. Bacteriophage application for biocontrolling Shigella flexneri in contaminated foods. J. Food Sci. Technol. 55:550-559.

23. Shahin, K., M. Bouzari, and R. Wang. 2018. Isolation, characterization and genomic analysis of a novel lytic bacteriophage vB_SsoS-ISF002 infecting Shigella sonnei and Shigella flexneri. J. Med. Microbiol. 67:376-386.

24. Sambrook, J., and D.W. Russell. 2001. Molecular Cloning: A Laboratory Manual. Cold Spring Harbor Laboratory, New York, NY.

25. Kropinski, A.M., A. Mazzocco, T.E. Waddell, E. Lingohr, and R.P. Johnson. 2009. Enumeration of bacteriophages by double agar overlay plaque assay. Methods Mol. Biol. 501: 69-76.

26. Viazis, S., M. Akhtar, J. Feirtag, A. Brabban, and F. DiezGonzalez. 2011. Isolation and characterization of lytic bacteriophages against enterohaemorrhagic Escherichia coli. J. Appl. Microbiol. 110:1323-1331.

27. Sack, D.A., C. Lyke, C. McLaughlin, and V. Suwanvanichkij. 2001. Antimicrobial Resistance in Shigellosis, Cholera and Campylobacteriosis. World Health Organization, Geneva.

28. Wittebole, X., S. De Roock, and S.M. Opal. 2014. A historical overview of bacteriophage therapy as an alternative to antibiotics for the treatment of bacterial pathogens. Virulence 5:226-235.

29. Nüesch-Inderbinen, M., N. Heini, K. Zurfluh, D. Althaus, H. Hächler, and R. Stephan. 2016. Shigella antimicrobial drug resistance mechanisms, 2004-2014. Emerg. Infect. Dis. 22:1083-1085.

30. Ghosh, S., G. Pazhani, G. Chowdhury, S. Guin, S. Dutta, K. Rajendran, M. Bhattacharya, Y. Takeda, S. Niyogi, and G.B. Nair. 2011. Genetic characteristics and changing antimicrobial resistance among Shigella spp. isolated from hospitalized diarrhoeal patients in Kolkata, India. J. Med. Microbiol. 60:1460-1466.

31. Williams, P.C., and J.A. Berkley. 2018. Guidelines for the treatment of dysentery (shigellosis): a systematic review of the evidence. Paediatr. Int. Child Health 38:S50-S65.

32. Mandomando, I., D. Jaintilal, M.J. Pons, X. Vallès, M. Espasa, L. Mensa, B. Sigaúque, S. Sanz, J. Sacarlal, and E. Macete. 2009. Antimicrobial susceptibility and mechanisms of resistance in Shigella and Salmonella isolates from children under five years of age with diarrhea in rural Mozambique. Antimicrob. Agents Chemother. 53:24502454.

33. Beladi Ghannadi, S., M. Ghane, and L. Babaeekhou. 2019. Determination of antibiotic resistance pattern and fre- quency of CTX-M, TEM, and SHV B-lactamase encoding genes among Shigella isolates from inpatients in Tehran, Iran. Med. Lab. J. 13:8-15.

34. Hartman, A.B., I.I. Essiet, D.W. Isenbarger, and L.E. Lindler. 2003. Epidemiology of tetracycline resistance determinants in Shigella spp. and enteroinvasive Escherichia coli: characterization and dissemination of tet (A)-1. J. Clin. Microbiol. 41:1023-1032.

35. Sow, A.G., A. Aïdara-Kane, O. Barraud, M. Gatet, F. Denis, and M.-C. Ploy. 2010. High prevalence of trimethoprimresistance cassettes in class 1 and 2 integrons in Senegalese Shigella spp isolates. J. Infect. Dev. Ctries. 4:207-212.

36. Barrantes, K., and R. Achí. 2016. The importance of integrons for development and propagation of resistance in Shigella: the case of Latin America. Br. J. Microbiol. 47: 800-806.

37. Baker, K.S., T.J. Dallman, N. Field, T. Childs, H. Mitchell, M. Day, F.-X. Weill, S. Lefèvre, M. Tourdjman, and G. Hughes. 2018. Horizontal antimicrobial resistance transfer drives epidemics of multiple Shigella species. Nat. Commun. 9:1462.

38. San Millan, A. 2018. Evolution of plasmid-mediated antibiotic resistance in the clinical context. Trends Microbiol. 26:978-985.

39. Lopatkin, A.J., H.R. Meredith, J.K. Srimani, C. Pfeiffer, R. Durrett, and L. You. 2017. Persistence and reversal of plasmidmediated antibiotic resistance. Nat. Commun. 8:1689.

40. Andersson, D.I., and D. Hughes. 2011. Persistence of antibiotic resistance in bacterial populations. FEMS Microbiol. Rev. 35:901-911.

41. Shahin, K., M. Bouzari, and R. Wang. 2019. Complete genome sequence analysis of a lytic Shigella flexneri vB_SflS-ISF001 bacteriophage. Turk. J. Biol. 43:99-112.

42. Mai, V., M. Ukhanova, M.K. Reinhard, M. Li, and A. Sulakvelidze. 2015. Bacteriophage administration significantly reduces Shigella colonization and shedding by Shigellachallenged mice without deleterious side effects and distortions in the gut microbiota. Bacteriophage 5:e1088124.

43. Bernasconi, O.J., V. Donà, R. Tinguely, and A. Endimiani. 2018. In vitro activity of 3 commercial bacteriophage cocktails against Salmonella and Shigella spp. Isolates of human origin. Pathog. Immun. 3:72-81.

Address correspondence to: Majid Bouzari, DVM, DipVSt, PhD

Department of Biology

Faculty of Sciences

University of Isfahan

Hezar Jereeb Street, 81746-73441

Isfahan

Iran

E-mail: bouzari@sci.ui.ac.ir

Ran Wang, PhD

State Key Laboratory Cultivation Base of MOST Institute of Food Safety and Nutrition Jiangsu Academy of Agricultural Sciences Nanjing 210014

P.R. China

E-mail: ranwang@jaas.ac.cn 